\title{
Indian Journal of Gastroenterology July-August 2020
}

\author{
Jimmy K. Limdi ${ }^{1}$ (D) \\ Published online: 5 October 2020 \\ (C) Indian Society of Gastroenterology 2020
}

\section{Apoptosis in peripheral blood lymphocytes in intestinal tuberculosis and Crohn's disease: Implications to diagnostic differentiation}

Differentiating intestinal tuberculosis (ITb) from Crohn's disease $(\mathrm{CD})$ poses real challenges to clinicians, not only in countries with a high prevalence, but also globally, as a result of immigration and risks conferred by immunosuppressive medications in vulnerable groups [1]. Unsatisfactorily low sensitivities of tissue staining, culture and polymerase chain reaction (PCR) pose a further layer of complexity, and whilst empirical anti-TB therapy has side effects, delays in treatment of CD may be associated with complications [2]. Nayak and colleagues [3] studied the differences between apoptosis in peripheral lymphocytes in ITb and CD. CD4+ cells as a percentage of the lymphocytes and viable, dead, total apoptotic, early apoptotic, and late apoptotic CD4+ cells were assessed in the peripheral blood by flow cytometry in healthy controls and patients with confirmed active ITb and CD prior to initiating therapy. The percentage of CD4+ cells in the peripheral blood was low in ITb but not different in CD compared with controls. These findings deserve further validation studies.

\section{Utility of narrow-band imaging in predicting histology in celiac disease}

The incidence and prevalence of celiac disease $(\mathrm{CD})$ in Asia are increasing [4]. The diagnosis involves clinical suspicion supported by positive serology and diagnostic endoscopy with

Jimmy K. Limdi

Jimmy.Limdi@nhs.net

1 Division of Gastroenterology, The Pennine Acute Hospitals NHS Trust, Manchester Academic Health Sciences, University of Manchester, Manchester, UK histological assessment of the duodenal mucosa. White light endoscopy (WLE) may not detect endoscopic features of villous atrophy (scalloping, grooving and mosaicism) resulting in an opportunity to diagnose the disease if biopsies are taken from unaffected mucosa [4].

Sinha and colleagues [5] report an experience of using narrow-band imaging with magnification (NBI-ME) in a prospective observational study in 122 patients with suspected CD (symptoms and elevated tTG antibodies) and 40 controls. The sensitivity and specificity of NBIME in predicting villous atrophy were $95.5 \%$ and $90 \%$, respectively, with good intra-observer agreement $(\kappa=$ 0.72 ). NBI-ME combined with $>5$-fold elevated tTG showed sensitivity and specificity for villous atrophy of $100 \%$ and $87.5 \%$, respectivly. Further studies across a wide range of serologies comparing WLE, NBI-ME and histology are needed to validate these findings.

\section{Predictors of immediate and short-term mortality in spontaneous bacterial peritonitis}

Spontaneous bacterial peritonitis (SBP) is an ominous complication of cirrhosis, a marker of end-stage liver disease and indication for liver transplantation (LT) [6]. Patients with cirrhosis and ascites are at risk of SBP with $1.5 \%$ to $3.5 \%$ prevalence in outpatients (where it may be asymptomatic) and $10 \%$ in hospitalized patients. Earlier diagnosis and treatment have reduced mortality to $20 \%$ and may enable selection of patients suitable for LT [6].

Sanglodkar and colleagues [7] report their experience of 122 patients with SBP from a cohort of 610 with cirrhosis and ascites. Fifty-two patients died, 40 survived beyond 3 months without LT and 30 underwent a LT within 3 months of diagnosis. Blood culture positivity (26.2\%) was associated with immediate mortality at 3 months. The investigators suggest intensive management in this group. Better diagnostic techniques for early SBP diagnosis are urgently needed to 
facilitate treatment and early identification of patients who could benefit from LT.

\section{Early, late or no shunt embolization in patients with cirrhosis and portosystemic shunt-related hepatic encephalopathy}

Portal hypertension (PHT) is a serious complication of chronic liver disease, and the development of portosystemic shunts (PSS) with antecedent complications portends an ominous prognosis. Philips and colleagues [8] retrospectively analyzed clinical outcomes, liver disease severity and survival between patients undergoing early shunt embolization (ESE) $(n=22)$ vs. late shunt embolization (LSE) $(n=23)$ and compared ESE with matched historical controls not undergoing shunt embolization and followed them for 18 months. Arterial ammonia, total bilirubin and Child-Pugh scores were significantly higher at baseline in the LSE group, which also had a higher postprocedure length of intensive care unit stay, infection rate and recurrence of encephalopathy in the first 9 months as also PHT-related adverse events beyond 10 months, compared with the ESE group. Mortality was significantly higher in the LSE group (60.8\% vs. $18.2 \%)$. Prospective studies factoring clinical variables are needed to validate ESE for large PSS in patients with cirrhosis.

\section{Relapse rate following withdrawal of anti-TNF therapy in patients with inflammatory bowel disease: A real-life cohort from northern India}

Despite their established role in inflammatory bowel disease treatment, the question of anti-(tumor necrosis factor [TNF]) withdrawal continues to intrigue. Among biological and disease-specific factors, the variable risk of infection and rare (but real) risk of lymphoproliferative and skin cancer deserve consideration [9]. Sahu and colleagues [10] report a retrospective experience of anti-TNF withdrawal from 79 patients. Thirty-three of 45 patients discontinued therapy while on clinical remission. Treatment was discontinued following loss of response in 11 , adverse effects $(\mathrm{TB}=10)$ and financial constraints in 15 patients. Of 15 patients who relapsed, 6 each responded to steroid or antibiotics and 3 patients each restarted anti-TNF or had surgery. Despite inherent limitations from the sample size, disease heterogeneity, inclusion of nonresponders, adverse effects and "clinical" remission as bases of drug withdrawal, this study highlights real challenges posed by cost and indeed risk of TB re-activation in a vulnerable population. Prospective studies in well-characterized cohorts factoring deep remission and drug levels among other biological predictors are urgently needed.

\section{Prevalence of low bone mineral density in inflammatory bowel disease and factors associated with it}

A myriad of factors are associated with low bone mineral density (BMD) in inflammatory bowel disease (IBD), including an active inflammatory state, calcium and vitamin D malabsorption, corticosteroid usage and reduction in physical activity [11]. Khan et al. [12] studied the prevalence of low BMD in a cohort of 106 IBD patients using radial quantitative ultrasound and compared it with age and gender-matched controls. Although they noted no differences in BMD between IBD patients and controls, serum calcium and vitamin D levels were lower in IBD patients. Older age ( $>50$ years) and longer disease duration were associated with significantly low BMD, whilst steroid usage at least once negatively predicted low BMD. Larger studies more inclusive of all age groups with a range of disease activity are needed. Meanwhile, steroid "stewardship", tight control of disease activity and control of other risk factors for osteopenia cannot be overemphasized as indeed periodic BMD assessment in line with national guidelines.

\section{Quantifying risk factors for ischemic colitis: a nationwide, retrospective cohort study}

Ischemic colitis (IC) is a cause of significant morbidity and mortality in individuals with predisposing factors and particularly in individuals over the age of 60 years [13]. Early identification may reduce the risk of mortality and indeed reduce morbidity from prolonged hospitalization and complications. Twohig et al. from USA [14] report on a populationbased retrospective study using the International Business Machines Exploris database that aims to quantify the risk of diseases and medications associated with IC. Of 1560 patients with IC from a database of 57 million, they identified hyperlipidemia as having the highest association followed by hypertension, congestive cardiac failure, constipation, prior abdominal surgery and atrial fibrillation. Additionally, they noted that beta blockers and pro-inflammatory conditions (vasculitis, rheumatoid arthritis and malignancy) also increased risk of IC. These are interesting and important findings that should raise an index of suspicion for IC in context, when early or timely intervention may be effective.

\section{Serological markers and molecular analysis of hepatitis B infection in a tertiary care hospital at Kathmandu, Nepal}

Hepatitis B is a major cause of viral hepatitis and related complications with associated mortality. Although acute and 
chronic hepatitis $\mathrm{B}$ are well recognized, occult infection (HBsAg negative, HBV DNA $<200 \mathrm{IU} / \mathrm{mL}$ ) is a risk factor for disease progression. Shrestha and colleagues [15] investigated the prevalence of serological markers, genotype, subgenotype and phylogenetic analysis of viral hepatitis in Nepal. Four sub-genotypes (A1, C1, D1 and D5) were detected with a higher prevalence in males $(65.6 \%)$ and nearly a third each in those aged 19-28 and 29-38 years of age which is concerning. Furthermore, $9 \%$ of samples showed occult infection which in terms of hidden burden of disease is concerning. That said, such studies are urgently needed and may serve as a launching point for prospective population screening mitigating against the morbidity and mortality from hepatitis B infection.

\section{Prevalence and determinants of hepatopulmonary syndrome in decompensated chronic liver disease}

Hepatopulmonary syndrome (HPS) characterized by gas exchange abnormalities and intrapulmonary vascular dilatation is a severe pulmonary vascular complication of chronic liver disease (CLD) and associated with significant mortality [6]. Liver transplantation (LT) is currently the only effective treatment. Severe hypoxia (Pao2 $<50 \mathrm{mmHg}$ ) may be associated with poor post-transplant prognosis, making early recognition and timely referral for LT crucial.

Khiangte and colleagues report a cross-sectional observational study to determine the prevalence and determinants of HPS in CLD [16]. HPS was diagnosed in 26 of 64 patients, consistent with previously reported literature. Spider naevi, clubbing, palmar erythema and orthodeoxia were significantly associated with the risk of HPS, although etiology of CLD was not. On multivariate analysis, a higher Model for Endstage Liver Disease (MELD)-Na score, hepatic encephalopathy and impaired diffusion capacity of lung for carbon monoxide (DLCO) were independently associated with risk of HPS [16]. A suitable index of suspicion taken together with adverse clinical parameters may facilitate earlier referral for LT as discussed by authors.

\section{Under-expression of microRNA-146a and 21 and their association with Crohn's disease}

The microRNAs (miRNAs) are a class of noncoding RNAs which regulate gene expression via translational repression or transcriptional degradation [17]. Dysregulation of miRNAs is implicated in the pathogenesis of inflammatory bowel disease (IBD). Mirzakhani and colleagues [18] studied their role in the pathogenesis of Crohn's disease (CD).

From $23 \mathrm{CD}$ patients and 30 healthy controls (HCs), miRNAs, including miR-21, miR-29a, miR-29b, miR-31,
miR-146a, miR-155, miR-181a and miR-181c, were evaluated via TaqMan MicroRNA Assays. Expression of miR-146a and miR-21 was significantly decreased in CD patients relative to HCs. The investigators found a negative correlation between miR-146a and Harvey-Bradshaw index (HBI) and a positive correlation between miR-21 and miR-29b and HBI. Under-expression of miR-146a and miR-21, critical for the regulatory function of regulatory $\mathrm{T}$ cells, was associated with CD. Dysregulated miRNAs have potential as novel, reliable and non-invasive biomarkers and therapeutic targets in IBD and have now entered the realms of phase 2 trials.

\section{Primary eosinophilic gastroenteritis: A case series from western India}

Eosinophilic gastroenteritis is an inflammatory disorder characterized by eosinophilic infiltration of the gastrointestinal tract. The stomach and small intestine are most commonly affected [19]. Mucosal affection is most common (57\% to $100 \%$ ) characterized by abdominal pain, nausea, vomiting, dyspepsia, diarrhea, malabsorption or protein-losing enteropathy. The muscular layer is involved in $30 \%$ to $70 \%$ of cases and may cause bowel wall thickening and intestinal obstruction but can present as an obstructing mass or intussusception. The serosal variant is least common, characterized by peritoneal irritation leading to eosinophilic ascites and peripheral eosinophilia, peritonitis, and perforation in most severe cases. The mainstay of treatment is steroids although leukotriene inhibitors, mast-cell stabilisers, immunomodulators and biological agents have been described in case series [19]. Jadhav et al. [20] report a case series describing variants of eosinophilic gastroenteritis, their diagnosis and treatment. An index of suspicion is necessary to make a diagnosis of eosinophilic gastroenteritis, which may be more common than we realize.

\section{Compliance with ethical standards}

Conflict of interest JKL declares that he has no conflict of interest.

Disclaimer The authors are solely responsible for the data and the contents of the paper. In no way, the Honorary Editor-in-Chief, Editorial Board Members, or the printer/publishers are responsible for the results/ findings and content of this article.

\section{References}

1. Limsrivilai J, Shreiner AB, Pongpaibul A, et al. Meta-analytic Bayesian model for differentiating intestinal tuberculosis from Crohn's disease. Am J Gastroenterol. 2017;112:415-27.

2. Torres J, Mehandru S, Colombel JF, Peyrin-Biroulet L. Crohn's disease. Lancet. 2017;389:1741-55.

3. Nayak SS, Shetty MV, Pai CG, et al. Apoptosis in peripheral blood lymphocytes in intestinal tuberculosis and Crohn's disease: 
implications to diagnostic differentiation. Indian J Gastroenterol. 2020;39. https://doi.org/10.1007/s12664-019-01011-z.

4. Singh P, Arora S, Singh A, et al. Prevalence of celiac disease in Asia: a systematic review and meta-analysis. J Gastroenterol Hepatol. 2016;31:1095-101.

5. Sinha SK, Berry N, Muktesh G, et al. Utility of narrow band imaging in predicting histology in celiac disease. Indian J Gastroenterol. 2020;39. https://doi.org/10.1007/s12664-020-01030-1.

6. European Association for the Study of the Liver. Electronic address eee, European Association for the Study of the L. EASL Clinical Practice Guidelines for the management of patients with decompensated cirrhosis. J Hepatol. 2018;69:406-60.

7. Sanglodkar U, Jain M, Venkataraman J. Predictors of immediate and short-term mortality in spontaneous bacterial peritonitis. Indian J Gastroenterol. 2020;39. https://doi.org/10.1007/s12664-02001040-z.

8. Philips CA, Rajesh S, George T, et al. Early, late, or no shunt embolization in patients with cirrhosis and portosystemic shuntrelated hepatic encephalopathy. Indian J Gastroenterol. 2020;39. https://doi.org/10.1007/s12664-020-01042-x.

9. Doherty G, Katsanos KH, Burisch J, et al. European Crohn's and Colitis Organisation topical review on treatment withdrawal ['Exit Strategies'] in inflammatory bowel disease. J Crohns Colitis. 2018;12:17-31.

10. Sahu P, Vuyyuru SK, Kante B, et al. Relapse rate following withdrawal of anti-TNF therapy in patients with inflammatory bowel disease: a real-life cohort from northern India. Indian J Gastroenterol. 2020;39. https://doi.org/10.1007/s12664-02001043-w.

11. Farraye FA, Melmed GY, Lichtenstein GR, Kane SV. ACG clinical guideline: preventive care in inflammatory bowel disease. Am J Gastroenterol. 2017;112:241-58.

12. Khan Z, Shetty S, Pai GC, et al. Prevalence of low bone mineral density in inflammatory bowel disease and factors associated with it. Indian J Gastroenterol. 2020;39. https://doi.org/10.1007/s12664020-01048-5.

13. Brandt LJ, Feuerstadt P, Longstreth GF, et al. ACG clinical guideline: epidemiology, risk factors, patterns of presentation, diagnosis, and management of colon ischemia (CI). Am J Gastroenterol. 2015;110:18-44 quiz 5.

14. Twohig PA, Desai A, Skeans J, Waghray N. Quantifying risk factors for ischemic colitis: a nationwide, retrospective cohort study. Indian J Gastroenterol. 2020;39. https://doi.org/10.1007/s12664020-01049-4.

15. Shrestha S, Mahatara S, Pun SB, et al. Serological markers and molecular analysis of hepatitis B infection in a tertiary care hospital at Kathmandu, Nepal. Indian J Gastroenterol. 2020;39. https://doi. org/10.1007/s12664-020-01051-w.

16. Khiangte B, Kothakota SR, Sasidharan M, et al. Prevalence and determinants of hepatopulmonary syndrome in decompensated chronic liver disease. Indian J Gastroenterol. 2020;39. https://doi. org/10.1007/s12664-020-01052-9.

17. Chapman CG, Pekow J. The emerging role of miRNAs in inflammatory bowel disease: a review. Ther Adv Gastroenterol. 2015;8:422.

18. Mirzakhani M, Khalili A, Shahbazi M, et al. Under-expression of microRNA-146a and 21 and their association with Crohn's disease. Indian J Gastroenterol. 2020;39. https://doi.org/10.1007/s12664020-01059-2.

19. Sunkara T, Rawla P, Yarlagadda KS, Gaduputi V. Eosinophilic gastroenteritis: diagnosis and clinical perspectives. Clin Exp Gastroenterol. 2019;12:239-53.

20. Jadhav R, Khairnar H, Kolhe K, et al. Primary eosinophilic gastroenteritis: a case series from western India. Indian J Gastroenterol. 2020;39. https://doi.org/10.1007/s12664-020-01067-2.

Publisher's note Springer Nature remains neutral with regard to jurisdictional claims in published maps and institutional affiliations. 